\title{
Disturbance-Observer-Based Robust Control of a Serial-link Robotic Manipulator Using SMC and PBC Techniques
}

\author{
Syed Ali AJWAD ${ }^{1}$, Jamshed IQBAL ${ }^{2 *}$, Abdul Attayyab KHAN ${ }^{3}$, Adeel MEHMOOD ${ }^{1}$ \\ ${ }^{1}$ Department of Electrical Engineering, \\ COMSATS Institution of Information Technology (CIIT), Islamabad, PAKISTAN \\ \{s.ajwad, adeel.mehmood\}@comsats.edu.pk \\ ${ }^{2}$ Department of Electrical Engineering, \\ National University of Computer and Emerging Sciences (FAST-NU), Islamabad, PAKISTAN \\ jamshed.iqbal@nu.edu.pk; \\ *Corresponding author \\ ${ }^{3}$ Dipartimento di Informatica, Bioingegneria, Robotica e Ingegneria dei Sistemi (DIBRIS), \\ University of Genova, ITALY \\ abdul.khan@edu.unige.it
}

\begin{abstract}
Robotic manipulators deployed in automation industry require high speed with precision and accuracy to perform sophisticated control tasks. Whereas, the factors like highly coupled dynamics, internal and external perturbation forces, joint friction and parameter variations degrade the performance of the manipulator. Consequently, the need of an advanced control technique or more preferably combination of multiple techniques with the capability of handling disturbances has been increased significantly. In the present research, design of Disturbance Observer (DO) based control techniques for a 6-Degree Of Freedom (DOF) robotic arm is presented to eliminate the effect of uncertainties and disturbances and to enhance the robustness of both Sliding Mode Control (SMC) and Passivity Based Control (PBC). Results demonstrate that the proposed controllers precisely estimate the torque yielded by external perturbation forces and improve the trajectory tracking performance of the system, which results in comparatively high performance of robotic manipulator in terms of speed and precision.
\end{abstract}

Keywords: Robot control, Robotic manipulator, Non-linear control, Industrial robot.

\section{Introduction}

Advancements in the field of automation have significantly accelerated the growth of manufacturing industry. Continuous assembly lines have been implemented to meet the requirement of mass production [1]. Robotic manipulators are considered as an integral part of such production lines as they offer potential advantages like accuracy, repeatability, throughput, swiftness, reliability and safety [2]. Furthermore, they can work in hazardous places where environment is harmful for human health [3]. Robots now perform various jobs ranging from simple task of pick and place to more sophisticated tasks like welding and cutting in automation industry [4]. Moreover, robots are also being extensively employed in the fields of medicine, defense, rescue and safety, mining, underwater and space exploration etc. [5]. Many industrial applications require fast and highly accurate motion of the robots. Precise movement of robotic links with high speed can only be achieved with a well-defined control strategy.
Control in robotics refers to the computation of input torque which ensures the accurate tracking of a desired trajectory. Research community has proposed various control strategies ranging from simple linear feedback control e.g. Proportional Integral Derivative (PID) to more sophisticated algorithms. A comprehensive review of such robust and nonlinear control strategies has been presented by Ajwad et al. in [6].

Sliding Mode Control (SMC), based on the variable structure control theory, lies in the category of non-linear robust control [7]. The advantages of SMC include system stability and robustness against matched uncertainties. Similarly, Passivity Based Control (PBC) is capable of handling the problems related with tracking and output feedback control. It employs the passivity property of physical system. The robot dynamics are complex and highly nonlinear in nature [8]. The position, velocity and acceleration of one joint have some affect over other joints. Furthermore, modeling uncertainties, friction, gravity and external forces can influence the motion of a 
robot as well. The performance of designed control technique can be enhanced significantly by adding a Disturbance Observer (DO) in feedback loop which can, substantially, reduce the aforementioned effects. It estimates variations in parameters and friction as external perturbations by using system dynamics.

Realization of control schemes based on DO in the domain of robotics is an active research area. DO has been utilized by Wang et al. in [9] to estimate the friction in a system and to eliminate external disturbances. Tracking performance of LuGre friction model-based motion controller has been improved by adding DO in the feedback loop. He and Xie have implemented DO to control a non-minimum phase system [10]. To compensate the nonlinearities and to cater noise in the feedback of system output, another controller comprising of high pass filter has been used. Chen et al. have designed a non-linear DO for 2- Degree Of Freedom (DOF) robotic manipulator in [11] which was further extended for a 3-DOF arm by Korayem and Haghighi in [12]. Stability of the proposed controller has been analyzed through Lyapunov's stability criterion. It has been demonstrated that non-linear DO based control structure provides superior performance even in the presence of friction and heavy payload. In [13], fast dynamics of tele-operated system, employed in master-slave configuration, has been controlled through DObased novel control technique. Parameters of the proposed controller determine the minimum rate of exponential convergence and also ensure the boundedness of tracking error even under fast-varying disturbances.
In the present work, DO is implemented to improve the performance of $1^{\text {st }}$ order SMC and PBC for a multi-DOF robotic arm. Both SMC and $\mathrm{PBC}$ are subjected to disturbance at input channel. It is shown that DO provides precise estimate of disturbances without any requirement of expensive force and torque sensors. The presence of DO greatly eliminates the effect of disturbances and increases the system robustness.

The remaining paper is organized as follows; Section 2 describes the manipulator system under study with a comprehensive mathematical model. Section 3 formulates SMC and PBC and presents the tracking results of both techniques. Design of DO and corresponding results have been detailed in Section 4. Finally, Section 5 comments on the conclusion of the paper.

\section{System Description}

In current research work, the system under consideration is a custom developed AUTonomous Articulated Robotic Educational Platform (AUTAREP) [14] shown in Figure 1. The platform is centered on a 6-DOF robotic manipulator which has five revolute joints from waist to wrist in an analogy to a human arm. The robotic arm consists of a two-fingered endeffector for object handling.

Joints of the robotic arm are actuated through precise DC servo motors (DME38B50G-11 for points and DME331337G-171 for endeffector). Optical encoders with each motor provide position feedback. The application areas of this manipulator include academia, research and automation industry [15].

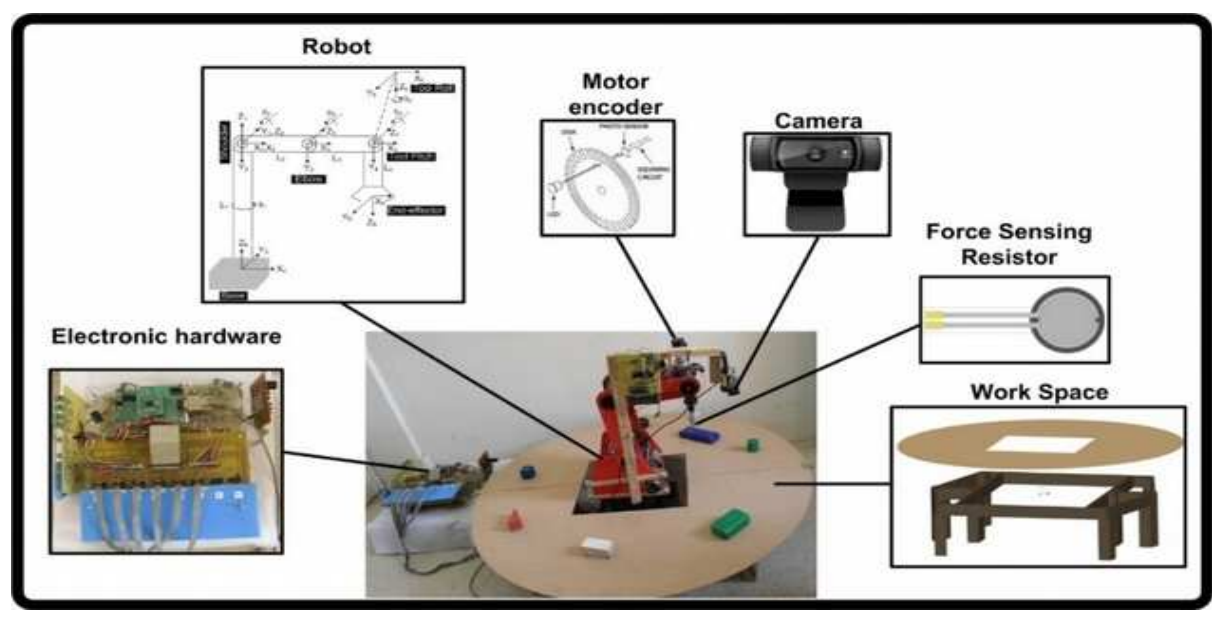

Figure 1. AUTAREP: A pseudo-industrial framework developed for research and educational purpose 
Design of the control algorithm demands deriving the mathematical model of the system. This can be attained by considering the robot kinematics and dynamics. Kinematics describes the relationship between robot joint angles and position and orientation of end-effector [16]. Denavit-Hartenberg (D-H) parameters based kinematic model of AUTAREP manipulator has been derived in [17]. Dynamic model expresses the motion of body in response to the forces/torques acting on it [18]. Various methods for the computation of dynamic model have been purposed by research community [19]. In current work, dynamic model of the manipulator has been derived using EulerLagrange method to simulate robot motion and to formulate control algorithm. Kinetic and potential energies of $i^{\text {th }}$ link can be computed (1) and (2) respectively.

$$
\begin{aligned}
& k_{i}=\frac{1}{2} m_{i} v_{c_{i}}^{T} v_{c_{i}}+\frac{1}{2}{ }_{i}^{i} \omega^{T i}{ }_{i} I_{i}^{i} \omega \\
& u_{i}=-m_{i} g^{T c}{ }_{i} P+u_{\text {refi }}
\end{aligned}
$$

where nomenclature is as follows:

- $\quad m_{i}$ is mass of the link

- $\quad v_{c_{i}}$ is linear velocity of the link w.r.t. Center of Mass (CoM)

- $\quad{ }_{i}^{i} \omega$ is angular velocity of the link with respect to its frame $i$

- $\quad{ }_{i}^{i} I$ is inertia of the link w.r.t. its frame $i$

- $\quad{ }_{i}^{c} P$ is CoM position of the link

- $\quad u_{\text {refi }}$ is reference potential energy of the link.

Lagrangian and torque of each link have been then computed using (3) and (4) respectively.

$$
L=k_{T}-u_{T}
$$

$\tau=\frac{d}{d t} \frac{\partial L}{\partial \dot{\theta}}-\frac{\partial L}{\partial \theta}$

where $k_{T}$ and $u_{T}$ represent total kinetic energy and potential energy related to each link respectively. The resulting dynamic model of the manipulator is represented by (5).

$$
\tau=M(q) \ddot{q}+V(q, \dot{q})+G(q)
$$

where $\tau$ denotes the input joint torque and $q$ represents the position of robot link. $M(q)$ describes the inertia matrix, $V(q, \dot{q})$ represents the matrix comprising of Corollis and Centrifugal forces and $G(q)$ is gravity matrix. The derivation details of $8^{\text {th }}$ order AUTAREP manipulator model is presented in [20].

\section{Control Design Techniques}

The derived dynamic model has been used to develop SMC and PBC control laws. The design detail of both laws is discussed below:

\subsection{Sliding mode control}

SMC can handle various classes of uncertain nonlinear systems especially in discrete domain with Multi-Inputs Multi-Outputs (MIMO) systems [21]. SMC provides insensitivity of the system to parameter variation, unknown joint inertia, friction and external disturbances. It consists of two phases generally known as reaching phase and sliding phase. The sliding phase begins within finite time when system trajectories, starting from their initial state, reach the sliding surface and evolve from there till all the further time. The block diagram of SMC is illustrated in Figure 2.

Consider a nonlinear system in canonical form, as given in (6).

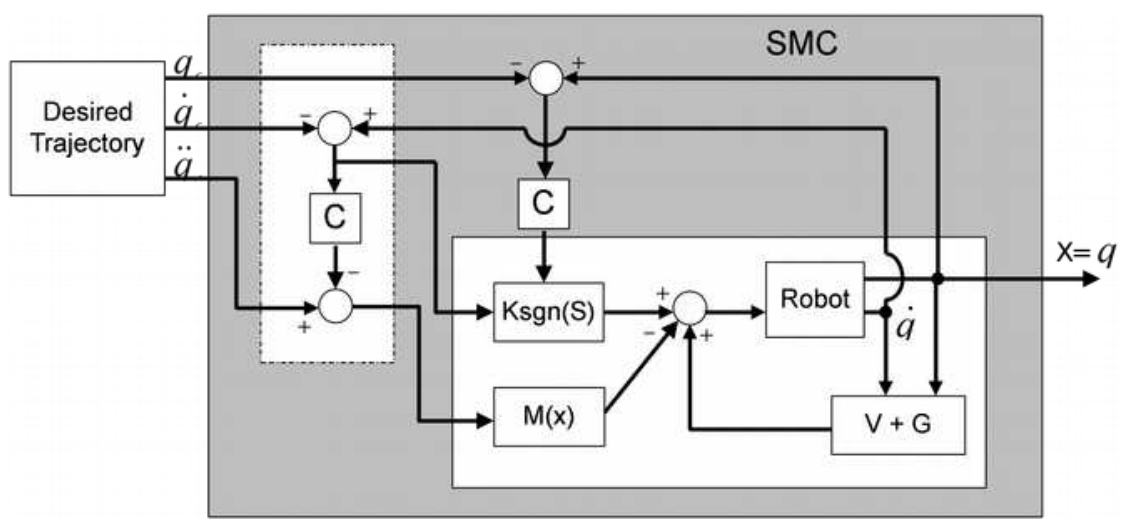

Figure 2. Block diagram of SMC 


$$
\dot{x}=f(x, t)+b(x, t) u+\Delta(x, t)
$$

where $x \in R^{n}$ and $u \in R^{m}$ denote the state vector and control input vector respectively.

The functions $f$ and $b$ are assumed to be continuous and smooth vector fields. $\Delta(x, t)$ is matched and bounded uncertainties with $\|\Delta(x, t)\| \leq D$. First step to design a SMC law is definition of sliding surface which follows from Hurwitz polynomial.

$$
S=\left(\frac{d}{d t}+C\right)^{r} x
$$

where $r$ is a constant which defines the order of the sliding mode controller. Sliding surface is typically chosen as (8)

$S=C x$

$C \in R^{1 \times n}$ is a row vector with compatible size to the state matrix $x \in R^{n \times 1}$. The objective in SMC is to force the sliding surface $S$ to zero through control law. This condition offers order reduction and the system evolved with $n-1$ states exhibits insensitivity w.r.t. the matched uncertainties. The controller designed through SMC consists of two parts as given in (9).

$$
u=u_{e q}+u_{d i s}
$$

where $u_{e q}$ represents equivalent controller [22] and $u_{d i s}$ is discontinuous part of controller. The equivalent control input can be obtained by replacing $\dot{S}=0$ along the system dynamics (9). For the sake of simplicity, assuming $\Delta(x, t)=0, u_{e q}$ is thus obtained as

$u_{e q}=\left(b^{-1}(x, t)\right)(-f(x, t))$

$u_{e q}$ drives the system from initial state to sliding surface and keeps $\dot{S}=0$. However, external disturbances and other uncertainties may cause $S \neq 0$. To handle this effect, $u_{d i s}$ is designed using a Lyapunov function (11).

$$
V=\frac{1}{2} S^{2}
$$

The time derivative of (11) along (6) will be

$$
\dot{V}=S\left(\frac{\partial S}{\partial x}\left(f(x, t)+b(x, t)\left(u_{e q}+u_{d i s}+\Delta(x, t)\right)\right)\right)
$$

Putting $u_{e q}$ from (10) in above expression, (12) is obtained.

$$
\dot{V}=S\left(b(x, t) u_{d i s}+\Delta(x, t)\right)
$$

Substituting $u_{\text {dis }}=-k \operatorname{Sign}(S)$, (12) becomes,
$\dot{V}=-S b(x, t)(k \operatorname{Sign}(s)-\Delta(x, t))$

$\dot{V} \leq-|S||b(x, t)||k-| \Delta(x, t) \mid)$

Using the bounded uncertainty condition, (13) becomes,

$$
\begin{aligned}
& \dot{V} \leq-|S||b(x, t)|(k-D) \\
& \dot{V} \leq-|S| \psi
\end{aligned}
$$

where $\psi>0$ for all $k>D$. Since for $u_{\text {dis }}=-k \operatorname{Sign}(S), \dot{V}$ becomes semi negativedefinite, the system dynamics converges to origin in finite time. In the present work, each link of the manipulator represents a second order system.

The control objective in this research is to ensure that the robot should track the predefined trajectory $q_{d}$. To achieve this objective, sliding surface based on error signal $\left(e=q_{d}-q\right)$ is defined by (14).

$$
S=\dot{e}+\lambda e+I \int e d t
$$

where $\lambda$ and $I$ are positive constants. The resulting control input torque following the design procedure is given by (15).

$$
\begin{aligned}
& \tau=M(q)\left[\lambda(\dot{e})+I(e)+\ddot{q}_{d}\right] \\
& +V(q, \dot{q})+G(q)-k \operatorname{sign}(S)-\zeta S
\end{aligned}
$$

The term $\zeta S$ has been introduced for strong reachability. The controlled torque guarantees the sliding mode and keeps the robot dynamics on sliding surface $S$ for all future values of time. Figure 3 depicts step response of base joint with corresponding torque.

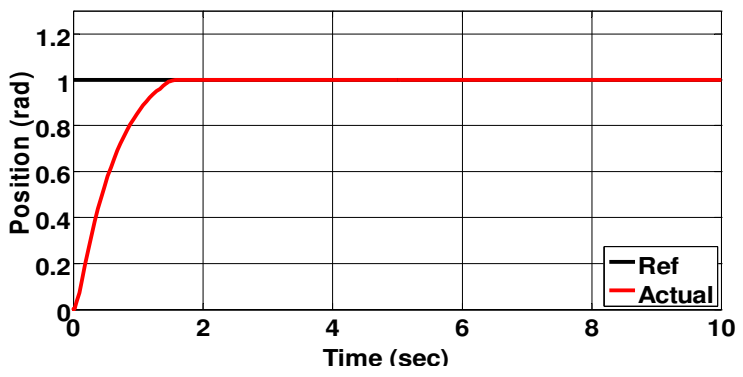

(a) Step response

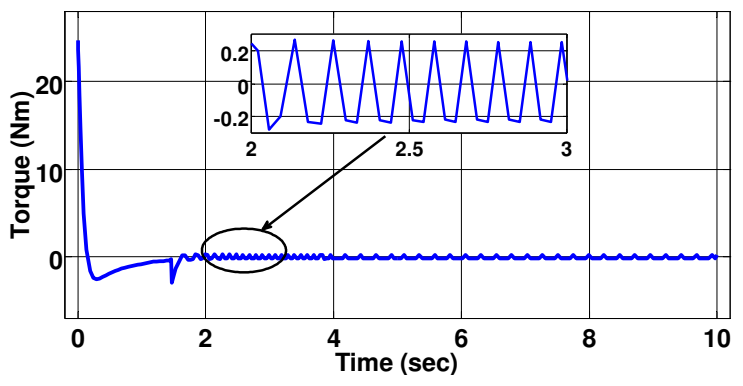

(b) Corresponding torque

Figure 3. Tracking performance of base joint 
Chattering is also visible in torque which is caused by discontinuous controller. Ramp and sinusoidal responses of elbow joint and wrist joints are illustrated in Figure 4(a) and (b) respectively. It can be observed that the control law is capable of tracking various kinds of reference trajectories.

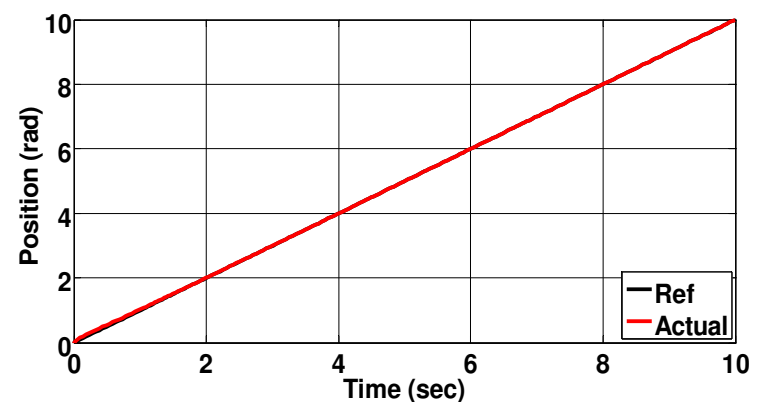

(a) Ramp response of elbow joint

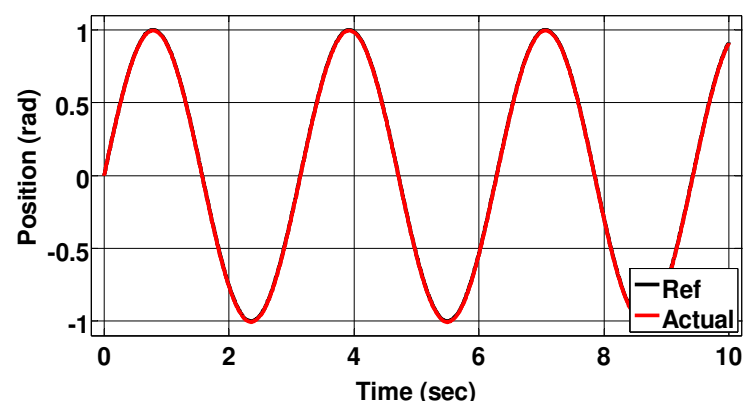

(b) Sinusoidal response of wrist joint

Figure 4. Tracking performance

\subsection{Passivity based control}

Passivity is a physical property of many systems and is related to energy dissipation that leads towards natural stability. Control laws, based on passivity property, are thus considered as energy reshaping and damping injection algorithms, which are suitable for both electrical and electromechanical systems. In manipulators, passivity from input torque to joint motion always exists and has been useful for designing passivity based controllers [23]. PBC law for trajectory tracking is given in (16).

$$
\tau=M(q) \ddot{q}_{r}+C(q, \dot{q}) \dot{q}_{r}+G(q)+v
$$

where $v$ is introduced for damping purpose and is given as

$$
v=-K_{d} s
$$

where $k_{d}$ is a positive gain diagonal matrix and $s$ is sliding surface defined by (18).

$$
s=\dot{q}-\dot{q}_{r}=\dot{e}+\lambda e
$$

where $e$ is the error matrix and $\lambda$ a positive gain diagonal matrix. The reference position of the robotic manipulator $q_{r}$ and its derivative are given by (19) and (20) respectively.

$q_{r}=q_{d}-\lambda \int_{0}^{t} e d t$

$\dot{q}_{r}=\dot{q}_{d}-\lambda e$

where $e$ is its desired position of the manipulator. The dynamics of robot manipulator can be reformulated as

$$
Y(q, \dot{q}) \hat{\theta}=M(q) \ddot{q}_{r}+C(q, \dot{q}) \dot{q}_{r}+G(q)
$$

where $Y(q, \dot{q}) \in R^{n \times p}$ is a regressor matrix and $\hat{\theta} \in R^{P \times 1}$ is a $p$-dimensional constant vector of the manipulator's physical parameters.

$$
\begin{aligned}
& \tau=Y(q, \dot{q}) \hat{\theta}-k_{d} s \\
& k_{d} s=\dot{\tau}+Y \widetilde{\theta}
\end{aligned}
$$

where $\widetilde{\theta}=\hat{\theta}-\theta$ and $\dot{\tau}=-[M(q) \dot{s}+C(q, \dot{q}) s]$. To demonstrate the passivity of the system, we need to show that $s, e \in L_{3}^{n} \cap L_{\infty}^{n}$. The error matrix $e$ is continuous and $e \rightarrow 0$ as $t \rightarrow \infty$. Therefore the error will asymptotically converge to zero [24].

Step response of shoulder joint with the corresponding torque is shown in Figure 5. It is worth observing that even after reaching the steady state, corresponding torque does not

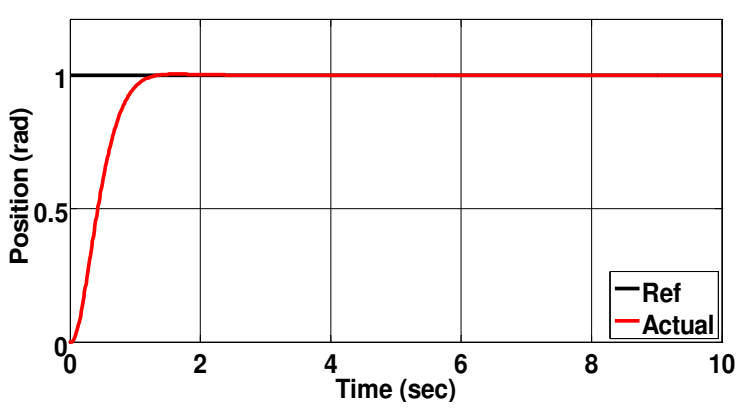

(a) Step response

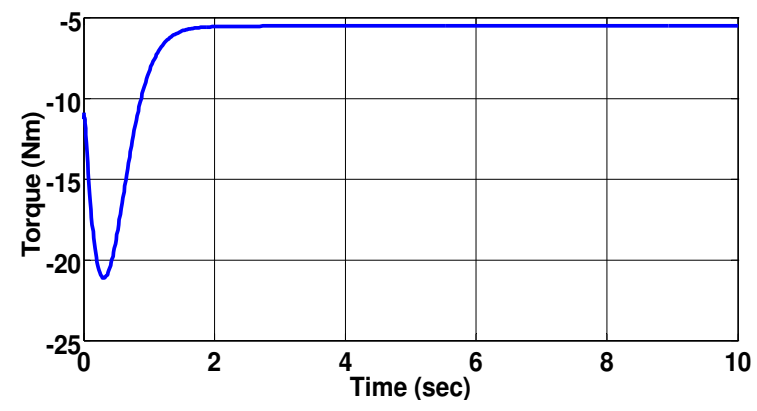

(b) Corresponding torque

Figure 5. Tracking performance of shoulder joint 
approach zero due to gravity effect. Tracking performance of elbow and wrist joints when respectively subjected to sinusoidal and ramp inputs respectively, are illustrated in Figure 6.

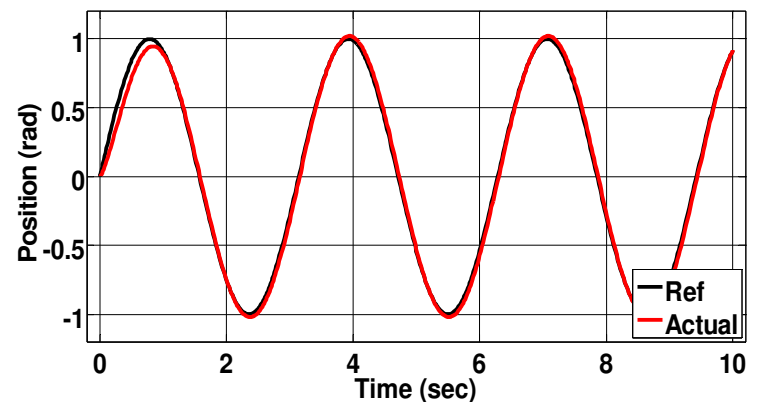

(a) Sinusoidal response of elbow joint

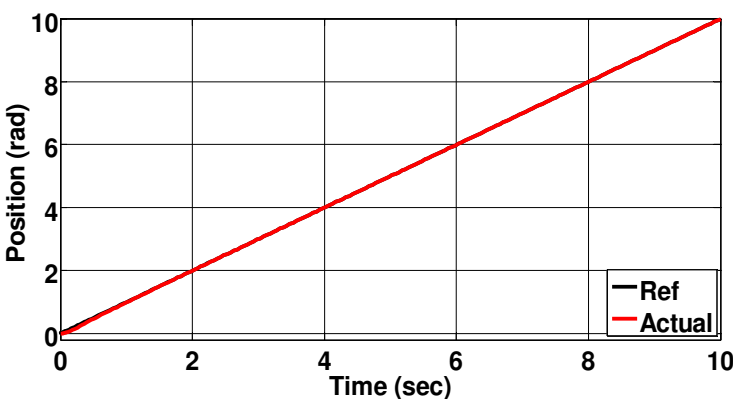

(b) Ramp response of wrist joint

Figure 6. Tracking performance

\section{Disturbance Observer Design}

The concept of DO based control was first introduced in 1980s [25]. In this control strategy, parameter variations, nonlinearities and friction are all dealt as disturbances along with the external disturbance that accumulates at the input channel. An estimator based on thesystem dynamics is designed to compute the disturbances thus eliminating requirement of external sensors. Each joint of the robotic manipulator can be controlled independently using this technique.
Dynamics of the robotic manipulator in the presence of external disturbance is given by (24).

$$
M(q) \ddot{q}+V(q, \dot{q})+g(q)+f(\dot{q})=\tau+\tau_{d}
$$

where $f(\dot{q})$ represents the friction forces and $\tau_{d}$ is the disturbance torque. The block diagram of DO based control for $\mathrm{i}^{\text {th }}$ link of a robotic arm is shown in Figure 7. Disturbance torque $\tau_{d}$ is estimated by designing an observer which takes system states i.e. position, velocity and acceleration as input and estimates the torque $\hat{\tau}$ given by (25).

$$
\hat{\tau}=\widehat{M}(q) \ddot{q}+\widehat{V}(q, \dot{q})+\hat{g}(q)
$$

This torque is then compared with input torque to get the disturbance term $\hat{\tau}_{d}$ (26), which is then passed through a low pass filter shown in the figure. Filter is designed so as to avoid the singularity issues that can appear in the system and to smoothen out the high frequency disturbance terms [26]. The cut-off frequency of the filter is chosen such that high frequency noises are rejected but the frequency of dynamics remains in the filter range.

$$
\hat{\tau}_{d}=\hat{\tau}-\tau
$$

\section{Discussion and Results}

For simulation purpose, disturbance has been added at input channel. Figure 8 shows the torque estimation due to disturbance. DO has been included in the feedback loop of both SMC and PBC laws. Figure 9 illustrates the system behavior in the presence of disturbance. SMC itself is a robust control technique but its performance may decrease due to high frequency and magnitude perturbation forces. Integration of DO with SMC has improved the controller ability in terms of robustness as can

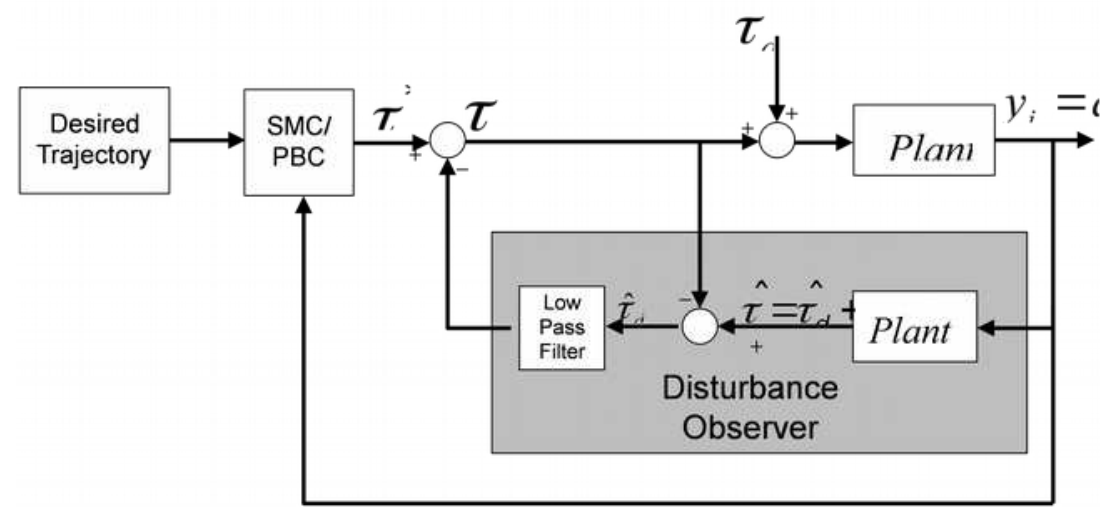

Figure 7. Block diagram of DO based control law 
be seen in Figure 9(a). In case of PBC, the capability of disturbance rejection has been increased significantly (Figure 9b) by the addition of DO. Table 1 illustrates a quantitative comparison of performance in term of error.

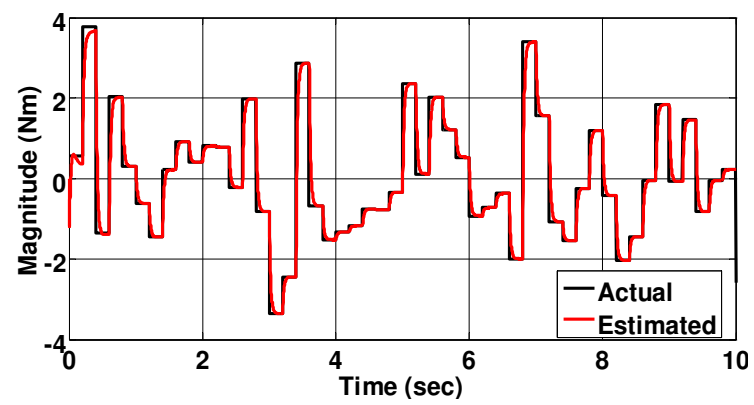

Figure 8. Disturbance torque estimation through DO

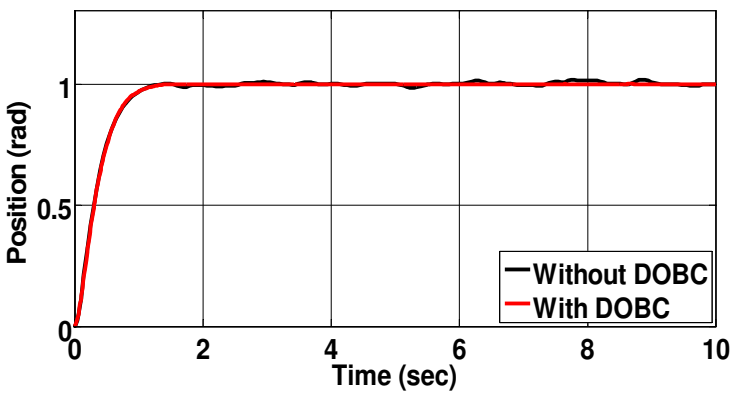

(a)

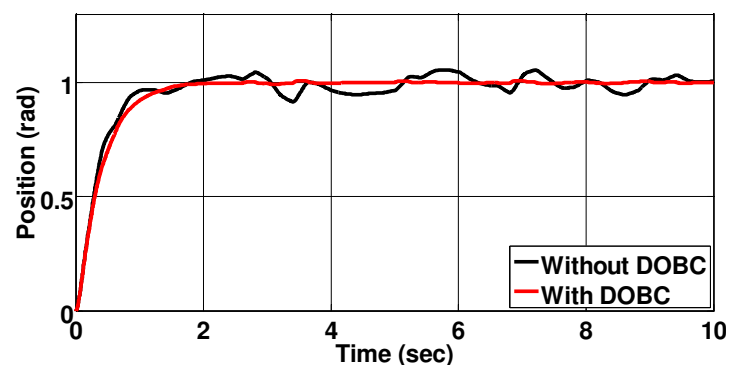

(b)

Figure 9. DO response with (a) SMC (b) PBC

Table 1. Performance comparison in term of error

\begin{tabular}{|c|c|c|}
\hline \multirow{2}{*}{$\begin{array}{c}\text { Control } \\
\text { Technique }\end{array}$} & \multicolumn{2}{|c|}{ \% error (max.) } \\
\cline { 2 - 3 } & $\begin{array}{c}\text { Without } \\
\text { DOBC }\end{array}$ & $\begin{array}{c}\text { With } \\
\text { DOBC }\end{array}$ \\
\hline SMC & 1.8 & 0 \\
\hline PBC & 8.4 & 0.8 \\
\hline
\end{tabular}

\section{Conclusion}

The paper presents a DO based control strategy to eradicate the disturbances which may affect the performance of serial-link robotic manipulator. DO has been used in feedback loop with both SMC and PBC separately. It has been shown that the observer approximates the disturbances in very precise manners and ensures stability of the system. Although SMC itself is a robust control technique but the use of DO further improves its performance by estimating the unwanted torques acting on the robot. Hardware implementation of DO on the actual AUTAREP framework is considered as a future work.

\section{REFERENCES}

1. FLOREA, G., C. LUPU, D. POPESCU, Supervised Solutions for Precise Ratio Control: Applicability in Continuous Production Line, Studies in Informatics and Control, vol. 23(1), 2014, pp. 53-64.

2. IQBAL, J., R. U. ISLAM, R. U., NABI, A. TAHIR, Robotics for Nuclear Power Plants - Challenges and Future Perspectives, International Conference on Applied Robotics for the Power Industry (CARPI), 2012, pp. 151-156.

3. BAHR, B., Y. LI, M. NAJAFI, Design and Suction Cup Analysis of a Wall Climbing Robot, Computers \& Electrical Engineering, vol. 22(3), 1996, pp. 193-209.

4. AJWAD, S. A., J. IQBAL, Emerging Trends in Robotics - A Review from Applications Perspective, International Conference on Engineering and Emerging Technologies (ICEET), 2015 (in press).

5. AJWAD, S. A., J. IQBAL, Recent Advances and Applications of Tethered Robotic Systems, Science International, vol. 26, no. 5, 2014, pp. 2045-2051.

6. AJWAD, S. A., K. BAIZID, J. IQBAL, M. I. ULLAH, A Comprehensive State-ofthe-Art on Control of Industrial Articulated Eobots, Journal of the Balkan Tribological Association, vol. 20, no. 4, 2014, pp. 499-521.

7. IQBAL, J., R. U. ISLAM, M. F., KHAN, Control Strategies for Robotic Manipulators, IEEE International Conference on Robotics and Artificial Intelligence (ICRAI), 2012, pp. 26-33.

8. MAI, T., Y. WANG, Adaptive Position Tracking Control System based on Recurrent Fuzzy Wavelet Neural Networks for Robot Manipulators, Proceedings of the Institution of 
Mechanical Engineers, Part I: Journal of Systems and Control Engineering, 2014.

9. DING, H., Y. WANG, Z. XIONG, X. ZHU, Nonlinear Friction Compensation and Disturbance Observer for a Highspeed Motion Platform, IEEE International Conference on Robotics and Automation (ICRA), 2004, pp. 4515-4520.

10. HE, Z., W. XIE, Improved Disturbance Observer based Control Structure, Chinese Control and Decision Conference, 2009, pp. 1015-1020.

11. BALLANCE, D. J., W.-H. CHEN, P. J. GAWTHROP, P. J., J. O'REILLY, A Nonlinear Disturbance Observer for Robotic Manipulators, IEEE Transactions on Industrial Electronics, vol. 47, no. 4, 2000, pp. 932-938.

12. HAGHIGHI, R., M. H. KORAYEM, Nonlinear Disturbance Observer for Robot Manipulators in 3D Space, Intelligent Robotics and Applications, Springer, 2008, pp. 14-23.

13. MARQUEZ, H., A. MOHAMMADI, M. TAVAKOLI, Disturbance Observer-based Control of Non-linear Haptic Teleoperation Systems, IET Control Theory and Applications, vol. 5, no. 18, 2011, pp. 2063-2074.

14. IQBAL, J., U. IQBAL, Z. NISSA, A. SAMAD, Embedded Control System for AUTAREP-A Novel AUTonomous Articulated Robotic Educational Platform, Tehnički vjesnik, vol. 21, no. 6, 2014, pp. 1255-1261.

15. IQBAL, J., R. U. ISLAM, A. KHALID, S. KHAN, S. MANZOOR, An Autonomous Image-guided Robotic System Simulating Industrial Applications, International Conference on System of Systems Engineering, 2012, pp. 344-349.

16. AJWAD, S. A., J. IQBAL, R. U. ISLAM, M. I. ULLAH, Modeling Robotic Arms A Review and Derivation of Screw Theory Based Kinematics, International Conference on Engineering and Emerging Technologies 2014, pp. 98.

17. IQBAL, J., R. U. ISLAM, H. KHAN, Modeling and Analysis of a 6 DOF Robotic Arm Manipulator, Canadian
Journal on Electrical and Electronics Engineering, vol. 3(6), 2012, pp. 300-306.

18. CRAIG, J. J., Introduction to Robotics: Mechanics and Control, Pearson Prentice Hall: Upper Saddle River, 2005.

19. MURRAY, R. M., Z. LI, S. S. SASTRY, A Mathematical Introduction to Robotic Manipulation, CRC press, 1994.

20. IQBAL, J., R. U. ISLAM, A. KHALID, S. MANZOOR, A. SAMAD, An Opensource Multi-DOF Articulated Robotic Educational Platform for Autonomous Object Manipulation, Robotics And Computer-Integrated Manufacturing, vol. 30, no. 3, 2014, pp. 351-362.

21. EL BAGDOURI, M., S. LAGHROUCHE, A. MEHMOOD, Study of the Nonlinear Control Techniques for Single Acting vgt pneumatic actuator, The International Journal of Vehicle Design, vol. 60, no. 3, 2012, pp. 264-285.

22. UTKIN, V. I., Sliding Modes in Control and Optimization, Springer-Verlag Berlin, 1992.

23. CAMPA, R., J. MORENOVALENZUELA, V. SANTIBÁÑEZ, Model-based Control of a Class of Voltage-Driven Robot Manipulators with Non-passive Dynamics, Computers \& Electrical Engineering, vol. 39, no. 7, 2013, pp. 2086-2099.

24. BASAÑEZ, L., E. NUÑO, R. ORTEGA, Passivity-based Control for Bilateral Teleoperation: A Tutorial, Automatica, vol. 47, no. 3, 2011, pp. 485-495.

25. MIYACHI, K., M. NAKAO, K. OHNISHI, A Robust Decentralized Joint Control based on Interference Estimation, IEEE International Conference on Robotics and Automation. Proc., 1987, pp. 326-331.

26. KIM, Y., I. NOH, J. SEOK, S. WON, An Adaptive Disturbance Observer for a Two-link Robot Manipulator, Intl. Conference on Control, Automation and Systems, 2008, pp. 141-145. 